\title{
O PET-Saúde e sua Interlocução com o Pró-Saúde a Partir da Pesquisa: o Relato dessa Experiência
}

\author{
The Educational Program for Health Work \\ (PET-Saude) and its Dialogue with the \\ Pró-Saúde Program based on Research: a \\ Case Report
}

Lucimare Ferraz

PALAVRAS-CHAVE:

- PET-Saúde

- Pró-Saúde

- Pesquisa

KEYWORDS:

- PET-Health

- Pro-Health

- Research
Recebido em: 28/03/2010 Aprovado em: 19/11/2010

\section{RESUMO}

Este relato de experiência tem por objetivo apresentar a bem-sucedida estratégia de implementação e integração do curso de pesquisa em saúde promovido pelo Pró-Saúde no Programa de Educação pelo Trabalho para a Saúde - PET-Saúde. Participaram do curso tutores, preceptores e acadêmicos de Medicina, Enfermagem, Educação Física, Fisioterapia, Nutrição, Psicologia e Serviço Social da Universidade Comunitária da Região de Chapecó. Como resultado, foi obtido o desenvolvimento de 18 projetos de pesquisa no PET-Saúde no município de Chapecó/SC no ano de 2009. Conclui-se que esta experiência reitera a iniciativa do Pró-Saúde em motivar não somente a classe acadêmica, como também os profissionais da área da saúde, para a investigação científica capaz de produzir conhecimentos essenciais para intervenção na realidade em saúde.

This case report discusses the successful implementation and integration of the health research course conducted by Pró-Saúde in the Educational Program for Health Work (PET-Saúde). Participants in the course included tutors, preceptors, and students of medicine, nursing, physical education, physical therapy, nutrition, psychology, and social work at the Chapecó Regional Community University. The experience resulted in the development of 18 research projects in PET-Saúde in the municipality of Chapecó, Santa Catarina State, in the year 2009. The article concludes that this experience reiterates the Pró-Saúde initiative to motivate not only academia, but also frontline health professionals, to conduct scientific research capable of producing essential knowledge for intervening in health reality. 


\section{INTRODUÇÃO}

A partir do Termo de Cooperação e Assistência Técnica, firmado em 29 de maio de 2008 entre o Ministério da Saúde e o Ministério da Educação, com o objetivo de desenvolver ações de capacitação de recursos humanos da área da saúde, foi instituído o Programa de Educação pelo Trabalho para a Saúde - PET-Saúde, destinado a fomentar grupos de aprendizagem tutorial na Estratégia Saúde da Família. Segundo a portaria interministerial, o PET-Saúde constitui-se um instrumento para viabilizar programas de aperfeiçoamento e especialização em serviço dos profissionais da saúde, bem como de iniciação ao trabalho, estágios e vivências dirigidos aos estudantes da área, de acordo com as necessidades do Sistema Único de Saúde SUS. São objetivos do PET-Saúde: I) possibilitar que o Ministério da Saúde cumpra seu papel constitucional de ordenador da formação dos profissionais da saúde, por meio da indução e do apoio ao desenvolvimento dos processos formativos necessários em todo o País, de acordo com características sociais e regionais; II) estimular a formação de profissionais e docentes de elevada qualificação técnica, científica, tecnológica e acadêmica, bem como a atuação profissional pautada pelo espírito crítico, pela cidadania e pela função social da educação superior, orientados pelo princípio da indissociabilidade entre ensino, pesquisa e extensão, preconizado pelo Ministério da Educação; III) desenvolver atividades acadêmicas em padrões de qualidade de excelência, mediante grupos de aprendizagem tutorial de natureza coletiva e interdisciplinar; IV) contribuir para a implementação das Diretrizes Curriculares Nacionais dos cursos de graduação da área da saúde; V) contribuir para a formação de profissionais de saúde com perfil adequado às necessidades e às políticas de saúde do País; VI) sensibilizar e preparar profissionais da área para o adequado enfrentamento das diferentes realidades de vida e de saúde da população brasileira; VII) induzir o provimento e favorecer a fixação de profissionais capazes de promover a qualificação da atenção à saúde em todo o território nacional; e VIII) fomentar a articulação entre ensino e serviço na área da saúde.

Segundo os Ministérios, um projeto de estímulo à implantação do PET-Saúde foi o Programa Nacional de Reorientação da Formação Profissional em Saúde - Pró-Saúde, pois, tendo em vista as mudanças curriculares nos cursos incluídos neste programa, verifica-se que ele atua estimulando a formação de docentes com um novo perfil, mais adequado às necessidades do SUS e da Estratégia Saúde da Família. Além disso, o Pró-Saúde está possibilitando o desenvolvimento da integração ensino-serviço e capacitação pedagógica, possibilitando que os profissionais que desempenham atividades na área da Atenção Básica à Saúde possam orientar os estudantes de graduação, em que o serviço público de saúde é o principal cenário de prática.

O Pró-Saúde está desde 2004 desenvolvendo atividades pedagógicas em consonância com as diretrizes do SUS na região de Chapecó, no estado de Santa Catarina. Entre as atividades, encontra-se o curso de Metodologia em Pesquisa na Saúde Coletiva. Este curso de capacitação em pesquisa do Pró-Saúde oferece aos trabalhadores, acadêmicos e docentes da área da saúde elementos teóricos de epidemiologia e de abordagem de análise qualitativa em pesquisa, para a realização do diagnóstico do estado de saúde da população, assim como possibilita a construção de indicadores que subsidiem o planejamento e as avaliações das intervenções do cuidado integral em saúde. Essa atividade busca a consolidação da articulação do ensino e da pesquisa, nos princípios da interdisciplinaridade e das necessidades sociais dos territórios de atuação dos serviços de saúde.

Diante disso, e buscando desenvolver estratégias articuladas para a expansão dos objetivos dos programas PET-Saúde, no ano de 2009, foi decidido potencializar e fortalecer as ações que já estavam sendo desenvolvidas no Pró-Saúde, dentre as quais se destaca o curso de Capacitação em Pesquisa em Saúde Coletiva. Tal ação se sobressai devido ao fato de estar em consonância com a política de formação e desenvolvimento de recursos humanos para a saúde do PET-Saúde.

Em 2009, o objetivo geral do curso foi capacitar os estudantes e profissionais da área da saúde envolvidos no PET-Saúde para desempenharem o papel de investigadores e articuladores de ações e estratégicas para a resolução de problemas no âmbito da saúde coletiva. Os objetivos específicos da iniciativa foram: conhecer os principais conceitos envolvidos nas atividades de pesquisa na área da saúde; elaborar projetos de pesquisa e trabalhos científicos com caráter investigativo e crítico da realidade da saúde do município de Chapecó e região; analisar e interpretar os dados em pesquisa científica; estar apto a orientar e acompanhar o desenvolvimento de trabalhos acadêmicos científicos direcionados às temáticas da área da saúde; promover ações interinstitucionais e intersetoriais, integrando estratégias de trabalho no desenvolvimento de pesquisas e resolução de problemas no campo da saúde; e publicizar os conhecimentos fundamentados em evidências científicas, contribuindo para as análises e condutas de novos saberes e formas de atuação na saúde.

Todo o trabalho foi desenvolvido assentado na premissa teórica de que a pesquisa faz parte do processo de construção do conhecimento, contribuindo para o aprendizado do indivíduo que a realiza, pois é ele quem desenvolve as bases do método científico. Além disso, a sociedade e a comunidade 
beneficiam-se com a aplicação do novo conhecimento gerado pelos/para os indivíduos que a compõem. ${ }^{1}$

A pesquisa pressupõe conhecer o fenômeno de forma aprofundada, e o trabalho de campo apresenta uma possibilidade de aproximação com aquilo que se deseja conhecer e estudar - a partir das concepções teóricas que fundamentam o objeto da investigação. Para tanto, é necessário uma interação entre o pesquisador e os atores sociais envolvidos no trabalho. ${ }^{2}$ É importante assinalar que se acredita que a pesquisa deve inscrever uma perspectiva metodológica que valorize os processos de transformação da realidade, de enfrentamento de conflitos e de participação social. ${ }^{3}$

Segundo Pedro Demo, a prática da pesquisa tem demonstrado não só a importância de se fazer ciência, como também sua face formativa, educativa e emancipatória. Para o autor, a arte de saber pensar é, em grande parte, a arte da cidadania, e o cuidado metodológico não se encerra na lida científica, mas constitui profundamente o processo formativo de alunos, professores e profissionais. ${ }^{4}$

Com base nessas premissas, percebe-se que os objetivos do PET-Saúde e do Pró-Saúde consubstanciam-se no intuito de estimular a formação de profissionais e docentes de elevada qualificação técnica, científica, tecnológica e acadêmica, bem como a atuação profissional pautada pelo espírito crítico, pela cidadania e pela função social da educação superior, ambas orientadas pelo princípio da indissociabilidade entre ensino, pesquisa e extensão, preconizado pelo Ministério da Educação.

Esse relato de experiência tem por objetivo apresentar a bem-sucedida estratégia de implementação e integração do curso de Pesquisa em Saúde promovido pelo Pró-Saúde no Programa de Educação pelo Trabalho para a Saúde - PET-Saúde.

\section{METODOLOGIA}

Para implementar o curso, foram adotadas metodologias ativas, prevendo processos de apropriação e produção do conhecimento. O processo de aprendizagem utilizou-se de instrumentos pedagógicos promotores de habilidades e competências no contexto da própria prática profissional, a qual desafiou os sujeitos envolvidos a um processo dialético de apropriação e transformação da realidade.

O diálogo foi o instrumento principal na interação pedagógica por meio de diferentes canais de comunicação para acompanhar o processo vivenciado. A materialização dos pressupostos anteriormente descritos ocorreu mediante atividades como: leituras, reflexões e debates; dinâmicas de grupo e trabalhos individuais; aulas expositivas e dialogadas; elabo- ração e reelaboração de conceitos e propostas de atuação de forma grupal; análise e interpretação do cotidiano do espaço da prática, a partir das quais os resultados foram apresentados no seminário de socialização dos estudos realizados.

O curso de capacitação foi desenvolvido com momentos presenciais e de atividades teórico-práticas (dispersão) e teve, em todo o processo, a supervisão e orientação de tutores do PET-Saúde.

\section{Os participantes}

Participam do curso seis tutores, 36 preceptores e 108 acadêmicos bolsistas e voluntários dos seguintes cursos: Medicina, Enfermagem, Educação Física, Fisioterapia, Nutrição, Psicologia e Serviço Social.

\section{As atividades}

A seguir são apresentadas as atividades desenvolvidas pelos grupos PET-Saúde da Unochapecó, demonstrando essas ações à luz das diretrizes de monitoramento e avaliação do Programa, apontadas nos itens II a $\mathrm{X}$ a seguir:

II - Participação dos alunos dos grupos em atividades, projetos e programas de ensino, pesquisa e extensão no âmbito do PET-Saúde.

III - Desenvolvimento de novas práticas e experiências pedagógicas, contribuindo para a implementação das Diretrizes CurricularesNacionaisdoscursosdegraduaçãodaáreadasaúde. IV - Produção de conhecimento relevante na área da atenção básica em saúde no âmbito da Estratégia Saúde da Família.

V - Planos de pesquisa em consonância com as áreas estratégicas de atuação da Política Nacional de Atenção Básica em Saúde.

VI - Projetos que contemplem a interdisciplinaridade, a atuação multiprofissional e a integração ensino-serviço.

VII - Projetos que contemplem a interação com a comunidade.

VIII - Alinhamento das atividades dos grupos PET-Saúde a políticas públicas e de desenvolvimento na sua área de atuação (Pró-Saúde, Política Nacional de Educação Permanente em Saúde, Programa Telessaúde Brasil, Unasus, entre outras).

IX - Publicações e participações em eventos acadêmicos de professores tutores, preceptores e estudantes.

$X$ - Instituição e desenvolvimento de atividades no âmbito dos Núcleos de Excelência em Pesquisa Aplicada à Atenção Básica.

A primeira atividade desenvolvida foi a "Oficina de indicadores para o diagnóstico do Nasf", promovida pelo Pró-Saúde, que teve como objetivo fortalecer a capacidade dos diferentes atores envolvidos no PET-Saúde da Unochapecó para a tomada de decisões e o planejamento em saúde, com definição de indicadores de diagnóstico, acompanhamento e 
avaliação da situação da saúde, fortalecendo a parceria entre ensino, serviço e comunidade (diretrizes e indicadores II, III, IV e VIII).

Ao final dessa atividade, houve o encontro da coordenação do Pet-Saúde com os tutores para os últimos detalhes de organização das ações a serem desenvolvidas na primeira reunião de tutores, estudantes e preceptores. Esse encontro teve por objetivo planejar trabalhos focados nas novas práticas e experiências pedagógicas contemplando a interdisciplinaridade, estabelecendo uma ligação com os projetos do Pró-Saúde (diretrizes e indicadores III, VI e VIII).

No primeiro encontro oficial das atividades do PET-Saúde, inicialmente, houve a acolhida dos acadêmicos e preceptores pelos tutores no salão de atos da Universidade. Nesse momento, foi apresentado o projeto do Pró-Saúde e do PET-Saúde. Na sequência, o grande grupo foi dividido em seis, cada um com um tutor, seis preceptores e demais acadêmicos. Esses subgrupos se dirigiram às salas de aula, onde foram realizadas dinâmicas de apresentação e, em seguida, cada dois preceptores foram agregados a seis ou oito acadêmicos, que elegeram um relator e debateram quais seriam os conteúdos / conceitos necessários para compreender e planejar pesquisas/diagnósticos na atenção integral à saúde. Posteriormente, houve a socialização e síntese das discussões e definições daquela manhã de trabalho, a partir das quais ficou estabelecido que os grupos iriam estudar as seguintes temáticas: territorilização; SUS; Estratégia Saúde da Família; PET-Saúde; e metodologia de pesquisa em saúde (diretrizes e indicadores II, III, IV e VIII).

A partir das necessidades e interesses levantados pelos preceptores e acadêmicos, iniciou-se a leitura de materiais bibliográficos e normatizações que possibilitassem o aprofundamento das discussões sobre a importância do conhecimento do território e da compreensão do diagnóstico (clínico e social) para intervenção em saúde, bem como reflexões sobre a importância do Pet-Saúde na formação profissional que atenda às diretrizes do SUS e sua vinculação com o Pró-Saúde na Unochapecó (diretrizes e indicadores II, III e IV).

Concomitantemente aos trabalhos anteriormente descritos, foi desenvolvido junto aos preceptores e acadêmicos o curso, com duração de 128 horas, sobre Metodologia da Pesquisa em Saúde Coletiva, financiado pelo Pró-Saúde. A seguir, são apresentados os momentos desse processo.

Primeiro momento: foram realizadas leituras de material bibliográfico sobre os temas: o conceito de pesquisa e a relevância de pesquisar; pesquisa qualitativa; pesquisa quantitativa; a importância da pesquisa científica e os tipos de abordagem com o foco na construção do conhecimento. Como atividade de dispersão, o grupo de acadêmicos e preceptores pesquisaram sobre os sistemas de informação (SI) existentes nos serviços de saúde a que pertenciam. Nessa busca de informações, deveriam responder às seguintes questões: 1) Quais são os SI que eles alimentam?; 2) Quais são os SI que eles manipulam?; 3) Quais são os SI em que eles buscam dados?; e 4) Quais são as possibilidades e limitações dos SI em saúde que eles conhecem/têm acesso? No encontro seguinte com os tutores, essas questões foram debatidas e relativizadas.

Segundo momento: foi realizada uma aula expositiva para o grande grupo, com quatro horas de duração, ministrada por um dos tutores, sobre pesquisa de dados secundários; as fontes de informação em saúde; sistema de dados do SUS; a busca e seleção de artigos e teses nos principais bancos de dados em saúde. Como atividade de dispersão, o grupo fez a busca de artigos e/ou trabalhos sobre diagnóstico em saúde da comunidade.

Terceiro momento: os grupos apresentaram e discutiram os artigos selecionados na atividade de dispersão, debatendo sobre os tipos de pesquisas científicas e sua aplicabilidade na Estratégia Saúde da Família. Como atividade de dispersão, os grupos assistiram a um destes filmes: Cobaia (1997), com direção de Joseph Sargent; Quase Deus (2004), com direção de Joseph Sargent; e O óleo de Lorenzo (1992), com direção de George Miller. Esses filmes serviram para alimentar o debate sobre ética em pesquisa e ética profissional no trabalho em equipe multiprofissional em saúde, já que a ética era a temática do quarto momento.

Quarto momento: houve uma palestra ministrada pelo Coordenador do Comitê de Ética da Unochapecó sobre os princípios e aspectos éticos em pesquisa. O palestrante também expôs os procedimentos necessários para o encaminhamento de submissão de projetos de pesquisa ao Comitê. Como atividade de dispersão, foi solicitada a busca de estudos sobre o trabalho interdisciplinar e intervenções na Estratégia Saúde da Família.

Quinto momento: dois tutores realizaram uma apresentação ao grande grupo sobre como estruturar um projeto de pesquisa, destacando os tópicos: problema; objetivos; justificativa; e metodologia da pesquisa. Nessa atividade foi disponibilizado material (produzido pelos próprios tutores) sobre a elaboração do projeto de pesquisa. Como atividade de dispersão, os grupos se reuniram para definir (ou predefinir) o problema, os objetivos e a população para a qual o projeto seria direcionado.

Vale destacar que as temáticas e os problemas de pesquisa foram discutidos junto às equipes de saúde e, quando possível, com lideranças comunitárias. A razão disso encontra-se 
no fato de que o objetivo aqui era possibilitar a construção de um diagnóstico que possibilitasse visualizar as demandas de intervenção, as quais deverão ser implementadas no ano de 2010.

Sexto momento: os grupos apresentaram o tema, o problema, os objetivos e a população de seus estudos. Em seguida, cada grupo recebeu orientações específicas de seus tutores. Como atividade de dispersão, os grupos reviram/discutiram/ contemplaram as sugestões dos tutores, elaborando, assim, um projeto contendo introdução, justificativa, objetivos, referencial bibliográfico, metodologia, cronograma, orçamento e referências. Para essa etapa foram destinados, aproximadamente, 25 dias.

Sétimo momento: nesse dia os grupos trocaram entre si os seus projetos e, com base em um roteiro (elaborado por um dos tutores), avaliaram os projetos uns dos outros, apontando fragilidades a serem sanadas para a correção final. Os trabalhos nos pequenos grupos foram orientados pelos professores tutores. Como atividade de dispersão, foram feitas as adequações/alterações dos projetos, segundo sugestões dos grupos e tutores, e o seu respectivo encaminhamento ao Comitê de Ética da Universidade. Enquanto aguardavam o parecer do Comitê, foram desenvolvidos os estudos pilotos, para testar as técnicas e instrumentos a serem aplicados nas pesquisas.

Oitavo momento: após a aprovação do Comitê de Ética em Pesquisa, houve a execução dos projetos, sempre com acompanhamento e apoio dos tutores. Esse foi um momento de intenso trabalho para os tutores, pois além de monitorar e incentivar o trabalho de campo, cada projeto aplicava uma abordagem diferente, o que exigiu dos tutores a busca de um corpo de conhecimento que sustentasse/amparasse as técnicas de coleta, processamento, análise e discussão dos dados (diretrizes e indicadores II, III, IV, V, VI, VII e VIII).

A seguir, a relação dos projetos desenvolvidos pelo PET-Saúde e Pró-Saúde no município de Chapecó, Santa Catarina, no ano de 2009:

1 - Análise do consumo alimentar de adolescentes em uma escola no município de Chapecó, SC.

2 - Cidade saudável: uma estratégia para melhoria de uma área de risco do Centro de Saúde da Família do município de Chapecó, SC.

3 - Desenvolvimento de uma proposta de atenção integral no programa Hiperdia, em dois Centros de Saúde da Família do município de Chapecó, SC.

4 - Protocolo de atendimento na atenção básica: ações e condutas diante dos agravos causados por acidentes com aracnídeos.
5 - Perfil epidemiológico dos usuários encaminhados ao Caps II pelos Centros de Saúde de Chapecó, SC, no primeiro semestre de 2009, com o diagnóstico de transtorno depressivo.

6 - Perfil do usuário encaminhado ao serviço de reabilitação pela rede básica de saúde no município de Chapecó, SC, com doenças osteomusculares.

7 - Impactos da implantação da proposta assistencial da classificação de risco do pronto-atendimento Efapi nos atores envolvidos nesse processo.

8 - Percepção das crianças moradoras de área de risco ambiental sobre as relações entre saúde e condições socioambientais no bairro Eldorado, Chapecó, SC.

9 - Elaboração de um protocolo para prevenção dos acidentes causados por aranhas do gênero loxosceles (arachnida aranae) na atuação da Estratégia Saúde da Família (ESF) em Chapecó, SC.

10 - A conscientização da população de um Centro de Saúde da Família no município de Chapecó, SC, em relação ao destino do lixo doméstico.

11 - Aspectos da saúde reprodutiva das gestantes do Centro de Saúde São Pedro - Chapecó, SC.

12 - Prevalência de distúrbios psiquiátricos em menores dentre os usuários de um Centro de Saúde da Família.

13 - O processo do risco nutricional entre crianças menores de um ano de idade assistidas no Centro de Saúde São Pedro, Chapecó, SC.

14 - A satisfação dos trabalhadores da Estratégia Saúde da Família atuantes em quatro diferentes Centros de Saúde da Família no município de Chapecó, SC.

15 - Aleitamento materno no primeiro ano de vida versus orientações recebidas durante o pré-natal no Centro de Saúde da Família Leste no município de Chapecó, SC.

16 - Prevalência de cáries em crianças de 5 e 6 anos de idade pertencentes à área de abrangência do CSF do bairro Seminário, no município de Chapecó, SC.

17 - Sentimentos e dificuldades vivenciadas pelo cuidador de crianças e/ou adolescentes usuários de álcool e/ou outras drogas atendidas na Caps I e II.

18 - Avaliação do estresse ocupacional em trabalhadores de uma equipe de Saúde da Família de Chapecó, SC.

Todos os projetos foram orientados e supervisionados pelos tutores, que tiveram a responsabilidade de proporcionar a apreensão dos elementos e ferramentas pelos preceptores e acadêmicos, para o desenvolvimento das pesquisas. Além de auxiliá-los no planejamento para a coleta de dados, os tutores também contribuíram no processo de análise e discussão dos resultados. 
Nono momento: houve a socialização dos resultados da pesquisa no dia 18 de dezembro de 2009. Nesse dia, estavam presentes os acadêmicos, preceptores, tutores, trabalhadores de saúde da secretaria municipal de saúde e docentes envolvidos nos projetos do Pró-Saúde. Foram apresentados resultados parciais dos projetos, bem como a sua discussão, junto à comunidade participante do seminário, com o intuito de pensar/propor ações de intervenção sobre a realidade estudada (diretriz e indicador IX).

Décimo momento: a construção do relatório de pesquisa e publicização dos seus resultados. Para essa atividade, foi convidada uma professora que proferiu uma palestra intitulada "Redação científica", na qual se apresentou os elementos que um texto acadêmico/científico deve ou não conter (diretriz e indicador IX).

\section{CONSIDERAÇÕES FINAIS}

Os programas Pet-Saúde e Pró-Saúde visam à mudança na formação profissional na área da saúde. Desse modo, as ações desenvolvidas devem resultar em um perfil profissional com habilidades para identificar, minorar e/ou resolver os problemas de saúde.

É nessa perspectiva que a conexão das ações dos dois programas se justifica e se referencia, pois o trabalho desenvolvido pelos grupos de acadêmicos, preceptores e tutores do PET-Saúde, com o apoio do Pró-Saúde, possibilitou o cumprimento das diretrizes propostas nos dois programas, as quais propõem a aplicação de tecnologias que promovam a saúde no âmbito da Estratégia Saúde da Família.

Nesse sentido, o exercício da pesquisa permitiu aos acadêmicos e profissionais da saúde desenvolverem atividades de caráter interdisciplinar, sendo um dispositivo para a troca de informações e saberes, além de fortalecer o vínculo e o acesso entre os serviços de saúde e a Universidade.

Outrossim, durante o desenvolvimento dos projetos de pesquisa, houve a oportunidade, a partir da revisão bibliográfica, de atualização de conceitos e aquisição de novos conhecimentos sobre as problemáticas investigadas, ratificando que os problemas de saúde devem ser estudados e resolvidos à luz da teoria em consonância com os saberes populares.
Com os resultados das pesquisas, ficou evidente para o grupo o quanto a investigação é importante para identificar as reais necessidades, os recursos e os indicadores de avaliação dos impactos das ações em saúde. Isso confirmou o pressuposto de que, sem dados/informações, o planejamento em saúde torna-se inconsistente, fadado a repetir ações infrutíferas, que não causam mudanças significativas no ambiente.

É possível concluir que essa experiência no PET-Saúde reitera a iniciativa do Pró-Saúde em motivar não somente a classe acadêmica, mas também os profissionais da área da saúde para a investigação, percebendo a pesquisa como fonte de produção coletiva de estratégias e ações de promoção da saúde em uma práxis contextualizada com as realidades local e cultural. Vale ressaltar que, apesar de alguns preceptores e acadêmicos mostrarem-se pouco envolvidos nas investigações, esses foram exceções, pois a maioria atuou de forma comprometida e entusiasmada, indo muitas vezes além do esperado pelos tutores, demonstrando-se preocupados e reflexivos em relação às suas próprias práticas.

\section{REFERÊNCIAS}

1. Clark OAC, Castro AA. A pesquisa. Pesqui Odontol Bras. 200317(Suppl 1):67-9.

2. Minayo MCS. Pesquisa Social: teoria, método e criatividade. Rio de Janeiro:Vozes; 1994.

3. Fernandes AD, Rozenowicz A, Ferreira JP. Avaliação qualitativa e indicadores sociais. 253. Psicol Estudo.2004;9(2):243-53.

4. Demo P. Cuidado metodológico: signo crucial da qualidade. SocEstado 2002;17(2):349-73

\section{CONFLITO DE INTERESSES}

Declarou não haver.

\section{ENDEREÇO PARA CORRESPONDÊNCIA}

Lucimare Ferraz

Rua Minas Gerais, 19 E, apto 802

Centro - Chapecó

CEP. 89801015 SC

E-mail: lferraz@unchapeco.edu.br 